\title{
Correction to: Exercise-induced circulating extracellular vesicles protect against cardiac ischemia-reperfusion injury
}

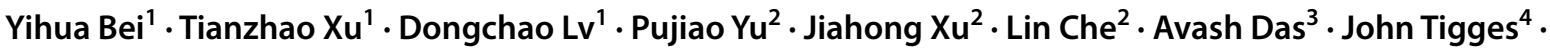 \\ Vassilios Toxavidis $^{4} \cdot$ Ionita Ghiran ${ }^{4} \cdot$ Ravi Shah $^{3} \cdot$ Yongqin $\mathrm{Li}^{1} \cdot$ Yuhui Zhang $^{5} \cdot$ Saumya Das $^{3} \cdot$ Junjie Xiao ${ }^{1}$
}

Published online: 8 October 2019

(c) Springer-Verlag GmbH Germany, part of Springer Nature 2019

\section{Correction to: Basic Res Cardiol (2017) 112:38 https://doi.org/10.1007/s00395-017-0628-z}

The original version of this article unfortunately contained a mistake. The protein band of internal control $\beta$-actin in Fig. 5c was duplicated as Fig. 3c, which was mistakenly presented (the samples for Figs. $5 \mathrm{c}$ and $3 \mathrm{c}$ were exactly the same). The statistical analysis for Fig. $5 \mathrm{c}$ was based on the phosphorylation level of ERK1/2 and HSP27 to total corresponding protein level. As the band of $\beta$-actin in Fig. 5c was not used in the statistical analysis, the correction of $\beta$-actin protein band will not influence the result and conclusion. We apologize for the inconvenience. Now the corrected blot for Fig. $5 \mathrm{c}$ is provided below. In addition, we would also like to add a note that Fig. 6a, c shared $\beta$-actin Western blot image, as these proteins were blotted on the same membrane. Also, the same case for Fig. 6b, d. The figure legend should note that samples for Fig. 6a, c; Fig. 6b, d were the same and these pairs of figures represent immunoblot from the same membrane (as $n=3$ per group). Hence they share the same beta-actin image. We apologize and these changes actually does not affect any data included in the article.

C

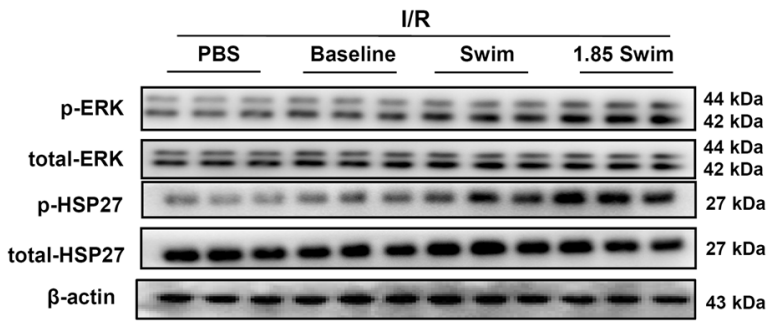

The original article can be found online at https://doi.org/10.1007/ s00395-017-0628-z.

Junjie Xiao

junjiexiao@live.cn

1 Cardiac Regeneration and Ageing Lab, School of Life Science, Shanghai University, 333 Nan Chen Road, Shanghai 200444, China

2 Department of Cardiology, Tongji Hospital, Tongji University School of Medicine, Shanghai 200065, China

3 Cardiovascular Division of the Massachusetts General Hospital and Harvard Medical School, Boston, MA 02114, USA

4 Beth Israel Deaconess Medical Center, Harvard Medical School, Boston, MA 02215, USA

5 Heart Failure Care Unit, Fuwai Hospital, State Key Laboratory of Cardiovascular Disease, National Center for Cardiovascular Diseases, Chinese Academy of Medical Sciences and Peking Union Medical College, Beijing 100037, China 